\title{
Massive Gravitons as Feebly Interacting Dark Matter Candidates
}

\author{
Haiying Cai®, ${ }^{1, *}$ Giacomo Cacciapaglia $\oplus^{2,3, \dagger}$ and Seung J. Lee $\odot^{1,+}$ \\ ${ }^{1}$ Department of Physics, Korea University, Seoul 136-713, Korea \\ ${ }^{2}$ University of Lyon, Université Claude Bernard Lyon 1, F-69001 Lyon, France \\ ${ }^{3}$ Institut de Physique des 2 Infinis de Lyon (IP2I), UMR5822, CNRS/IN2P3, F-69622 Villeurbanne Cedex, France
}

(Received 6 August 2021; accepted 1 February 2022; published 23 February 2022)

\begin{abstract}
We detail our discovery of a chiral enhancement in the production cross sections of massive spin-2 gravitons, below the electroweak symmetry breaking scale, that makes them ideal dark matter candidates for the freeze-in mechanism. The result is independent of the physics at high scales and points toward masses in the $\mathrm{keV}-\mathrm{MeV}$ range. The graviton is, therefore, a sub-MeV dark matter particle, as favored by the small scale galaxy structures. We apply the novel calculation to a Randall-Sundrum model with multiple branes, showing a significant parameter space where the first two massive gravitons saturate the dark matter relic density.
\end{abstract}

DOI: 10.1103/PhysRevLett.128.081806

Despite the overwhelming evidence for the presence of a dark matter (DM) component in our Universe, also indirectly observed in galaxies and galaxy clusters, the nature of this matter component remains a mystery. In the standard model (SM), no known particle can play the role of DM: the only candidates, neutrinos, have a relic density many orders of magnitude below the required one, which is roughly 5 times the relic density of ordinary baryons. Extended objects, like primordial black holes, remain a possibility, alas still requiring new physics to explain their presence [1].

A particle DM candidate can only emerge from new physics beyond the SM. The most popular and time honored possibility has been the weakly interactive massive particle (WIMP), which requires substantial interactions with the SM particles, with an annihilation cross section on the order of the electroweak ones, $\sigma v \simeq 2.0 \times 10^{-26} \mathrm{~cm}^{3} / \mathrm{s}$, independent of the DM mass in order to achieve the observed relic density $\Omega_{\mathrm{DM}} h^{2} \sim 0.12$ [2]. The nonobservation of new physics signals at colliders (the LHC) and at DM direct and indirect detection experiments has, however, put this scenario under stress [3]. Hence, this "WIMP crisis," has prompted the exploration of alternative possibilities. Here we will be interested in the freeze-in mechanism of feebly interacting massive particles (FIMPs), which never attain thermal equilibrium with the SM bath [4]. This is usually obtained by tuning a coupling to very small values, typically on the order of

Published by the American Physical Society under the terms of the Creative Commons Attribution 4.0 International license. Further distribution of this work must maintain attribution to the author(s) and the published article's title, journal citation, and DOI. Funded by SCOAP ${ }^{3}$.
$10^{-8}-10^{-10}$ provided the DM is stable (protected by a parity). Another possibility, called ultraviolet (UV) freezein [5], relies on higher-dimensional operators suppressed by a large scale. In the latter case, the naive expectation is that unitarity violation renders the DM relic density highly sensitive to the unknown reheat temperature at the end of the inflationary phase. Here, we will present a novel scenario based on a spin-2 DM candidate where, albeit the freeze-in is induced by a nonrenormalizable operator, the UV sensitivity of the predictions is curbed by the electroweak scale. The mechanism behind this is a chiral enhancement of some scattering amplitudes involving massive SM fermions, which is only activated below the electroweak symmetry breaking (EWSB) scale, $T_{\mathrm{EW}} \approx 160 \mathrm{GeV}$.

The couplings of the spin-2 state (also known as massive graviton) $G_{\mu \nu}$ to the SM particles can be parametrized by the following four-dimensional effective Lagrangian:

$$
\mathcal{L}_{\text {eff }}=\left.\sum_{i=\text { spin }} C_{i} G^{\mu \nu}\left(2 \frac{\delta \mathcal{L}^{i}}{\delta \hat{g}^{\mu \nu}}-\eta_{\mu \nu} \mathcal{L}^{i}\right)\right|_{\hat{g}=\eta},
$$

where $\eta$ is the Minkowski metric, and the factors within parentheses are the stress-energy tensors $T_{\mu \nu}^{i}$ for the SM particles of different spins $\left(i=0, \frac{1}{2}, 1\right)$. As such, $\mathcal{L}^{i}$ is the SM Lagrangian for the particles of spin $i$ and, since the spin sum of the graviton polarization $P_{\mu \nu, \alpha \beta}=\frac{1}{2}\left(P_{\mu \alpha} P_{\nu \beta}+P_{\nu \alpha} P_{\mu \beta}-\frac{2}{3} P_{\mu \nu} P_{\alpha \beta}\right), \quad$ with $\quad P_{\mu \nu}=$ $\eta_{\mu \nu}-\left(k_{\mu} k_{\nu} / M_{G}^{2}\right)$ is traceless, the terms proportional to $\mathcal{L}^{i}$ in Eq. (1) do not contribute. Explicit expressions for the various spins can be found in Ref. [6]. Note that the massive spin-2 DM candidate is not the mediator of gravitational interactions, which are generated by the usual massless gravitons in our scenario. Universal couplings 
between the massive graviton $G_{\mu \nu}$ and all the SM particles ensures unitarity in the high energy limit before the chiral symmetry breaking. Hence, we will stick to this assumption and denote the universal coupling as $C_{H}$. The results we present here are independent of the origin of the degrees of freedom needed to give mass to the spin-2 state, while later we will focus on a class of five-dimensional models, where the mass is generated by the compactification of the extra space.

The freeze-in generally proceeds via decays of heavier particles or pair annihilations of the SM particles in the thermal bath. The FIMP belongs to a hidden sector that communicates to the SM sector via a superweak portal. Assuming that the inflaton dominantly decays into the SM particles, the initial DM abundance after reheating can be neglected, so that the FIMP relic density is produced via an accumulation process. In our simplified model, the massive graviton is a perfect FIMP candidate for DM due to the smallness of the gravitational coupling $C_{H}$. As we will see, however, as the massive graviton can decay via the same coupling, $C_{H}$ is forced to be too small to produce a sizable density of gravitons. Here we will point out a mechanism in place below the EWSB scale that invalidates this conclusion. First, we introduce the Boltzmann equation (BE) describing the production of the FIMP graviton in the early Universe. For a generic scattering process $B_{1}+B_{2} \rightarrow B_{3}+G$, where $B_{1,2,3}$ are the SM particles, the evolution of the massive graviton number density $n_{G}$ follows the BE [4],

$$
\begin{aligned}
\frac{d n_{G}}{d t}+3 H n_{G} \approx & \frac{T}{512 \pi^{6}} \int_{s_{0}}^{\infty} d s P_{B_{1} B_{2}} P_{B_{3} G} \\
& \times \mathcal{A}_{B_{1} B_{2} \rightarrow B_{3} G} K_{1}(\sqrt{s} / T) / \sqrt{s},
\end{aligned}
$$

where $H$ is the Hubble expansion parameter defined as the time derivative of the logarithmic scale factor $\ln a(t), K_{1}$ is the first modified Bessel function of the second kind, and

$$
\begin{aligned}
P_{B_{i} B_{j}} & =\frac{\left[s-\left(m_{B_{i}}+m_{B_{j}}\right)^{2}\right]^{\frac{1}{2}}\left[s-\left(m_{B_{i}}-m_{B_{j}}\right)^{2}\right]^{\frac{1}{2}}}{2 \sqrt{s}}, \\
\mathcal{A}_{B_{1} B_{2} \rightarrow B_{3} G} & =\int d \Omega|\mathcal{M}|_{B_{1} B_{2} \rightarrow B_{3} G}^{2}, \\
s_{0} & =\max \left\{\left(m_{B_{1}}+m_{B_{2}}\right)^{2},\left(m_{B_{3}}+m_{G}\right)^{2}\right\},
\end{aligned}
$$

with $\mathcal{A}_{B_{1} B_{2} \rightarrow B_{3} G}$ standing for the amplitude squared after the solid angle integration and $s=\left(p_{B_{1}}+p_{B_{2}}\right)^{2}$. The righthand side of Eq. (2) is the interaction rate density $\gamma(T)$ for $\mathrm{DM}$ production, where the thermal average is performed by the technique developed in [7]. By solving the BE with the proper initial condition, one finds that the FIMP relic density is directly proportional to the $2 \rightarrow 2$ cross section, in contrast to the inverse proportionality in freeze-out models. At high temperatures, above the EWSB scale, we find that all amplitudes squared scale like $\mathcal{A}_{B_{1} B_{2} \rightarrow B_{3} G^{(1)}} \sim$ $C_{H}^{2} g_{i}^{2} s$, where $g_{i}$ is an appropriate SM coupling. The only exception is the process $h h \rightarrow h G$, for which the amplitude is a constant $\mathcal{A}_{h h \rightarrow h G} \propto C_{H}^{2}\left(m_{h}^{4} / v^{2}\right)$. As $C_{H} \sim M_{\mathrm{Pl}}^{-1}$ is suppressed by the Planck mass to ensure Hubble timescale stability, the cross sections are too small to provide a DM-like relic density via $2 \rightarrow 2$ scattering freeze-in.

In this Letter, we discovered a chiral enhancement of a class of processes that is active below the EWSB scale and in the limit of light graviton. Thus, in the following, we will assume that $M_{G}$ is much smaller than any other scale in the process. The chirally enhanced processes involve SM fermions and a massless gauge boson. The most dominant ones, therefore, involve quarks and gluons: $q \bar{q} \rightarrow g G$, $q g \rightarrow q G$, etc. Note that the process with heavy lepton pairs and one photon will also undergo this chiral enhancement, but suppressed by a factor of $e^{2} /\left(4 g_{s}^{2}\right)$ in the amplitude squared. For the process $\bar{q} q \rightarrow g G$, the total amplitude squared before EWSB is calculated (see the Supplemental Material [8]) using the Feynman Rules in $[6,9]$,

$$
\mathcal{A}_{\bar{q} q}^{0}=\frac{128 \pi}{3} C_{H}^{2} g_{s}^{2} s
$$

where $g_{s}$ is the chromodynamic coupling and the result is consistent with the results in [10] in the limit of $M_{G} \rightarrow 0$. In contrast, after EWSB, the leading term in the small $M_{G}$ expansion is given by

$$
\mathcal{A}_{\bar{q} q}=\frac{256 \pi C_{H}^{2} g_{s}^{2} m_{q}^{2} s\left(s+2 m_{q}^{2}\right)}{9 M_{G}^{4}} .
$$

This term comes from the contribution of the longitudinal polarization of the massive graviton in terms of $\left(k_{\mu} k_{\nu} / M_{G}^{2}\right)$, which contributes only after a chirality flip of the fermion line via a mass insertion. The enhancement is more effective for heavy quarks (the bottom and charm, as the top is too heavy to be in thermal equilibrium below the EWSB) and can overcome the Planck suppression for $M_{G}$ in the $\mathrm{keV}-\mathrm{MeV}$ range. For the process $q g \rightarrow q G$, we can use the cross symmetry to get the amplitude squared,

$$
\mathcal{A}_{q g}=\mathcal{A}_{\bar{q} g}=\frac{256 \pi C_{H}^{2} g_{s}^{2} m_{q}^{2}\left(s-m_{q}^{2}\right)^{2}\left(s+m_{q}^{2}\right)}{3 s M_{G}^{4}} .
$$

Note that, when inserting the amplitudes squared in the BE (2), we need to consider the renormalization group evolution of the coupling constant, $\alpha_{s}(\mu)=\left(g_{s}^{2} / 4 \pi\right)$ where $\mu=\sqrt{s}$. In the numerical results, we will use the following one-loop running coupling: 


$$
\frac{1}{\alpha_{s}(\mu)}=8.47+\frac{7}{2 \pi} \log \left(\frac{\mu}{M_{Z}}\right) .
$$

We are now ready to compute the massive graviton relic density. After reheating, the Universe entered into the radiation-dominated era, with the reheating temperature $T_{\mathrm{RH}}$ representing the maximum temperature reached by the thermal bath $[11,12]$. The freeze-in production of the massive graviton can be divided into two phases: the UV phase above the EWSB scale and the infrared (IR) one after EWSB, i.e., for temperatures above and below the critical temperature $T_{C} \simeq 160 \mathrm{GeV}$ [13]. The SM particles are in thermal equilibrium after reheating, while the massive graviton is not. This is because the superweak portal ensures $\gamma(T)<H n_{\gamma}^{\mathrm{eq}}$ both in the UV and IR phases. As a simple estimation, after EWSB, for the massive graviton to stay in nonequilibrium requires $T^{3} \lesssim \pi^{6} M_{\mathrm{Pl}}\left(M_{G}^{4} / m_{q}^{2}\right)$. Provided the FIMP mass is $M_{G} \sim$ $1 \mathrm{MeV}$, this translates to an upper bound $T_{C} \lesssim 1 \mathrm{TeV}$. Rewriting the BE in terms of the yield $Y_{G}=n_{G} / S$, with $S$ being the entropy density, the IR contribution to the freezein density can be written as

$$
\begin{aligned}
Y_{\mathrm{IR}} \simeq & \frac{1}{2048 \pi^{6}} \int_{T_{\mathrm{QCD}}}^{T_{C}} \frac{d T}{S H}\left[\int_{4 m_{q}^{2}}^{\infty} d s\left(s-4 m_{q}^{2}\right)^{1 / 2} A_{\bar{q} q} K_{1}\left(\frac{\sqrt{s}}{T}\right)\right. \\
& \left.+2 \int_{m_{q}^{2}}^{\infty} d s \frac{\left(s-m_{q}^{2}\right)^{2}}{s^{3 / 2}} A_{q g} K_{1}\left(\frac{\sqrt{s}}{T}\right)\right],
\end{aligned}
$$

with

$$
S=\frac{2 \pi^{2} g_{*}^{s} T^{3}}{45}, \quad H=\sqrt{\frac{g_{*}^{\rho} \pi^{2}}{90}} \frac{T^{2}}{M_{\mathrm{Pl}}}
$$

where $M_{\mathrm{Pl}}$ is the reduced Planck mass for the Hubble parameter in the radiation-dominated era, $T_{\mathrm{QCD}} \simeq$ $150 \mathrm{MeV}$ and $g_{*}^{s} \simeq g_{*}^{\rho} \simeq 10^{2}$. As typically $T_{\mathrm{RH}}>T_{C}$, the IR contribution is not sensitive to the reheating temperature $T_{\mathrm{RH}}$. Moreover, the temperature integration is dominated by the interval close to $T_{C}$ with the IR yield proportional to $m_{q}^{2}$. Hence, even though the processes involving lepton pairs can continue to temperatures below the QCD phase transition, their contribution remains subleading. By evaluating Eq. (8) numerically and taking into account the bottom and charm quarks, we obtain the following result:

$$
\begin{aligned}
\Omega_{\mathrm{IR}} h^{2} & =\frac{M_{G}}{3.6 \times 10^{-9} \mathrm{GeV}} Y_{\mathrm{IR}} \\
& \simeq 3.0 \times 10^{31} \mathrm{GeV}^{5} \frac{C_{H}^{2}}{M_{G}^{3}} .
\end{aligned}
$$

Since the couplings of $G$ to the SM particles are model independent and uniquely dictated by symmetries, we can ignore the self-interactions after including a radionlike field $r$. The decay width of a graviton of a few $\mathrm{MeV}$ mass is governed by the Lagrangian in Eq. (1),

$$
\Gamma\left(G \rightarrow e^{+} e^{-}+\nu_{i} \bar{\nu}_{i}+\gamma \gamma\right) \simeq \frac{9 C_{H}^{2} M_{G}^{3}}{320 \pi} .
$$

Now we can estimate the upper bound of the IR freeze-in contribution by combining Eq. (10) with the lifetime $\tau_{G}=$ $6.58 \times 10^{-25} / \Gamma_{G}$ from Eq. (11),

$$
\Omega_{\mathrm{IR}} h^{2} \lesssim 0.12 \times\left(\frac{1.6 \mathrm{MeV}}{M_{G}}\right)^{6} \frac{10^{27} \mathrm{sec}}{\tau_{G}} .
$$

Given the larger branching ratio of graviton into $\gamma \gamma$ than into $e^{+} e^{-}$, we impose an appropriate lifetime limit $\tau_{G} \gtrsim 10^{27} \mathrm{sec}$, deriving from the stringent bounds from indirect detection and cosmic microwave background (CMB) $[14,15]$. Hence, in Eq. (12), for each fixed value of $M_{G}$, the maximum relic density is given by the largest value of $C_{H}$ that satisfies the lifetime bound. We found, therefore, that the DM relic density can be saturated by a single massive graviton with $M_{G} \lesssim 1.6 \mathrm{MeV}$, with increasing lifetimes (decreasing $C_{H}$ ) for smaller masses.

The UV contribution can be computed with a similar formula to Eq. (8), taking into account all the quarks in the SM (which are massless in this regime). Numerically, we obtain

$$
\begin{aligned}
\Omega_{\mathrm{UV}} h^{2} & =1.2 \times 10^{29} \mathrm{GeV}_{H}^{2} M_{G} \\
& \simeq \Omega_{\mathrm{IR}} h^{2}\left(\frac{M_{G}}{4.0 \mathrm{GeV}}\right)^{4} .
\end{aligned}
$$

Note that we used $T_{\mathrm{RH}}=10^{6} \mathrm{GeV}$ as a template value [11] in the above estimate. Because of the linear divergence in $s$ of Eq. (4), the integration of BE gives $\Omega_{\mathrm{UV}} h^{2} \propto T_{\mathrm{RH}}$. One can anticipate, for $M_{G} \sim \mathcal{O}(1) \mathrm{MeV}$, that the UV result is at most $10^{-14}$ times the IR one and much smaller in the case of a lower $T_{\mathrm{RH}}$ input.

We now connect this result to a realistic model for the massive graviton. The model setup is an extension of the Randall-Sundrum (RS) model [16-18] with multiple branes [19-23], where all the SM particles are put on the intermediate brane $y=r_{1} \pi$ with a tension $T \propto\left(k_{2}-k_{1}\right)$, where $k_{1,2}$ are the curvatures in the two intervals. To estimate the relic density, we can ignore the small difference between the two curvatures and take $k \sim M_{\mathrm{Pl}}$. Hence, in addition to a tower of Kaluza-Klein (KK) massive gravitons $G^{(n)}$, the low energy theory contains a potentially light radion $r$ peaked around the IR brane $y=r_{2} \pi$. The effective Lagrangian in Eq. (1) needs to be extended with the interactions between the massive gravitons and the radion $r$ by expanding the RS metric, 


$$
\begin{aligned}
\mathcal{L}_{\text {eff }}= & C_{H} \sum_{i} G^{(n) \mu \nu} T_{\mu \nu}^{i}+d_{V} r V_{\mu \nu} V^{\mu \nu} \\
& +C_{r}\left(2 G^{(n) \mu \nu} G_{\mu \nu}^{0}-G_{\mu}^{(n) \mu} G_{\nu}^{0 \nu}\right) \square r \\
& +C_{\mathcal{Q}} \mathcal{Q}\left(G^{3}, G^{2} r, G r^{2}, r^{3}\right),
\end{aligned}
$$

where $\square r=\eta^{\mu \nu} \partial_{\mu} \partial_{\nu} r$ and $G^{0}$ is the massless graviton (zero mode). The last term in Eq. (14) is composed of cubic selfinteractions. The effective couplings for $G^{(n)}$ are derived by the integration of 5D wave function overlaps [24,25],

$$
\begin{gathered}
C_{H}=\frac{1}{\Lambda_{H}} \frac{x_{n}^{2}}{4 \sqrt{2} J_{2}\left(x_{n}\right)}, \\
C_{r}=\frac{1}{M_{\mathrm{Pl}}} \frac{\sqrt{6}\left[1-J_{0}\left(x_{n}\right)\right]}{x_{n}^{2} J_{2}\left(x_{n}\right)},
\end{gathered}
$$

where we applied an approximation in the limit of $e^{-k\left(r_{2}-r_{1}\right) \pi} \ll 1$ and defined $\Lambda_{H}=M_{\mathrm{Pl}} e^{-k\left(4 r_{1}-3 r_{2}\right) \pi}$. The $x_{n}$ is the root of $J_{1}\left(x_{n}\right)=0$. The self-interaction strength is characterized by $C_{\mathcal{Q}} p^{2}$ and the bulk integration predicts $C_{\mathcal{Q}} \sim 1 / \Lambda_{\mathrm{IR}}$ with $\Lambda_{\mathrm{IR}}=M_{\mathrm{Pl}} e^{-k r_{2} \pi}$. The coupling of radion to diphoton originates from the trace anomaly at the loop level,

$$
d_{\gamma}=\frac{\alpha_{\mathrm{EM}}}{8 \pi \sqrt{6} \Lambda_{r}}\left(b_{\mathrm{EM}}-\sum_{i} F_{i}\right)
$$

with the cutoff scale $\Lambda_{r}=M_{\mathrm{Pl}} e^{-k\left(2 r_{1}-r_{2}\right) \pi}$ and we used the electromagnetic beta function $b_{\mathrm{EM}}=-(11 / 3)$, with $\sum_{i} F_{i} \simeq-(1 / 9)$ related to the loop functions of $W$ and those heavy fermions including $t, b, c, s, \tau$, and $\mu[26,27]$.

The mass and coupling orders are of crucial importance for the cosmological stability of the lightest KK graviton as DM. Note that in this 5D model the coupling $G^{(1)}-G^{0}-G^{0}$ is highly suppressed by order of $M_{\mathrm{Pl}} e^{k r_{2} \pi}$, unlike the bigravity model, where this coupling is absent [28]. The decay width of a massive graviton into two zero gravitons is negligible. Also the hadronic decays are kinematically forbidden for $M_{G}$ in the $\mathrm{MeV}$ range. For the lightest $\mathrm{KK}$ graviton, due to the $\mathcal{O}(1)$ self-coupling, we have to require $m_{r}>M_{G^{(1)}} / 2$ to shut down the decay of $G^{(1)} \rightarrow 2 r$. Hence, the relevant decay channels are $G^{(1)} \rightarrow e^{+} e^{-}, \nu \bar{\nu}$, and $\gamma \gamma$ with their decay width given by Eq. (11). The other decay patterns will be determined by the mass of radion. Note that from naïve dimensional analysis a radion mass [or dilaton in the 4D conformal field theory (CFT) dual] is expected to be around the KK scale [29-31], without the necessity for a fine-tuning. First of all, we calculated the decay width for $G^{(n)} \rightarrow G^{0}+r$,

$$
\Gamma\left(G^{(n)} \rightarrow G^{0} r\right)=\frac{C_{r}^{2} m_{r}^{4}}{4 \pi M_{G}}\left(1-\frac{m_{r}^{2}}{M_{G}^{2}}\right),
$$

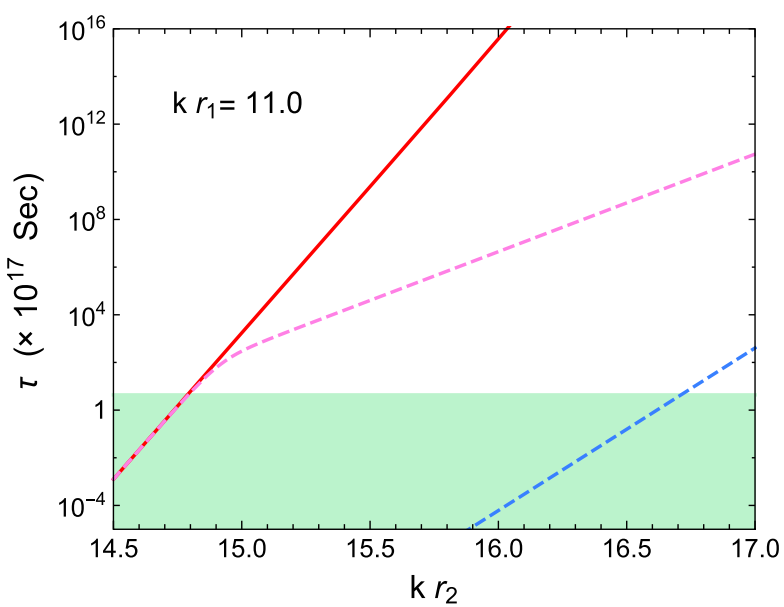

FIG. 1. The lifetime for the lightest KK graviton (red lines) and the IR brane radion (blue line) as a function of $k r_{2}$, with $k r_{1}=11.0$. The solid line corresponds to $m_{r}>2 m_{G}^{(1)}$, whereas the dashed line is for $m_{r}=3.45 \Lambda_{\mathrm{IR}}$. The region with lifetime below the age of the Universe $\left(\sim H_{0}^{-1}\right)$ is shaded in green.

with the other relevant decay widths of $G^{(n)} \rightarrow 2 G^{(1)}, 2 r$ and $r \rightarrow 2 G^{(1)}, 2 \gamma$ given in [32-34].

Here we consider two scenarios: in one case, $M_{G^{(1)}} / 2<$ $m_{r} \lesssim M_{G^{(2)}} / 2$ allows for a Planck-suppressed decay of $G^{(1)} \rightarrow G^{0}+r$ and a prompt decay of $G^{(2)} \rightarrow 2 r$; in the second, the radion is slightly heavier $m_{r} \gtrsim 2 M_{G^{(1)}}>M_{G^{(2)}}$, so that the radion and $G^{(n)}(n \geq 3)$ quickly decay into $2 G^{(1)}$ in less than $10^{-14} \mathrm{sec}$ for $M_{G^{(1)}} \sim 2 \mathrm{MeV}$. As shown in Fig. 1, for $k r_{1}=11.0$, we roughly need $k r_{2} \gtrsim 14.8$ to make the lowest KK graviton to be stable beyond the Hubble time level. However for the IR brane radion, a much larger value of $k r_{2} \gtrsim 16.7$ is required to barely ensure the same property. This reflects the fact that the cutoff scale $\Lambda_{H}$ of the KK graviton is of order Planck scale, while the one for the radion is smaller. For the lighter radion case, the dashed red line bends at a certain point, indicating that the dominant decay becomes $G^{(1)} \rightarrow G^{0}+r$, as the coupling $C_{r}$ does not decrease with the radius $r_{2}$ of the IR brane. A small $m_{r}$ will render the graviton $G^{(1)}$ less stable. Thus, the second scenario seems to be a more natural option, where the lightest two gravitons are both long-lived enough to play the role of DM.

In the following, we will focus on the heavier radion scenario. The heavier KK gravitons will also undergo a nonthermal IR freeze-in and afterward cascade deposit their density into the stable gravitons. Since the energy is conserved during the subsequent freeze-in, the relic density approximately is

$$
\Omega_{\mathrm{IR}} h^{2} \simeq 1.75 \times 10^{-62} e^{k\left(8 r_{1}-3 r_{2}\right) \pi} \sum_{n=1}^{10} x_{n}^{2},
$$

where we traded $\left(C_{H}, M_{G}\right)$ in Eq. (10) with the two radius parameters in the $5 \mathrm{D}$ realization and summed up all the 


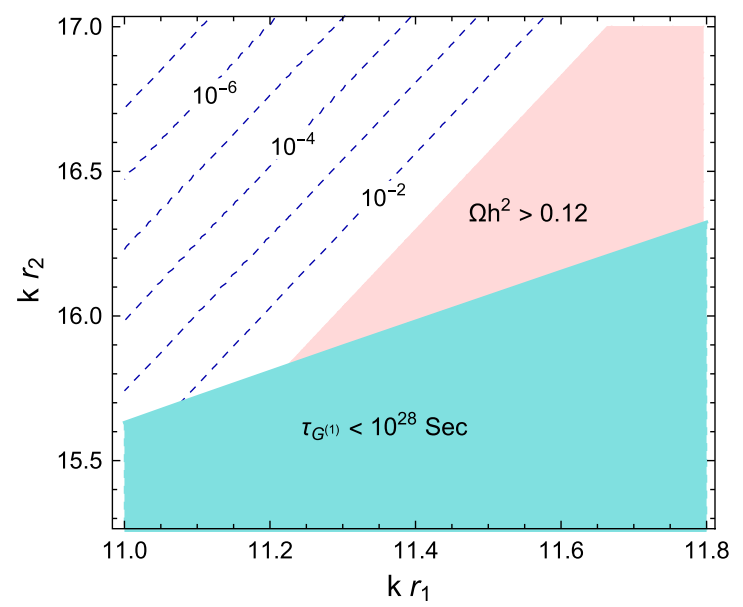

FIG. 2. The contour of relic density in the plane of $\left(k r_{1}, k r_{2}\right)$, where the blank region is viable for the graviton as long-lived DM. The boundary of the pink region satisfies $\Omega h^{2}=0.12$ by summing the direct and subsequent freeze-in. The light blue region is excluded by the indirect direction.

contributions until the $n=10 \mathrm{KK}$ graviton. Note that the self-interactions of the radion receive a very large (finite) one-loop correction from the KK gravitons, hence the 5D description will no longer be reliable beyond the $10 M_{\mathrm{KK}}$ scale. The prediction of Fig. 2 is consistent with the upper limit derived in Eq. (12). Since $G^{(1)}$ and $G^{(2)}$ mainly decay into diphoton and neutrinos, we impose a proper gamma-ray bound $\tau_{G^{(1)}} \sim 10 \tau_{G^{(2)}}>10^{28}$ sec for the viable relic density. In Fig. 2, the contours for relic density $\left(\Omega h^{2}=10^{-2}, 10^{-3}, 10^{-4}, 10^{-5}, 10^{-6}, 10^{-7}\right)$ are parallel to each other and equally spaced. Furthermore, the ratio of the lines is determined by the exponential factor in Eq. (19) $\left(\partial r_{2} / \partial r_{1}\right)=8 / 3$. For $k r_{1} \sim 11.2$ and $k r_{2} \sim 15.8$, the lowest two KK gravitons can achieve a large fraction of the observed DM relic density.

In summary, in this Letter we presented the effect of a chiral enhancement for the single production of a massive graviton in the early Universe. This enhancement is active below the EWSB scale and insensitive to the UV physics. Thanks to this novel effect, a generic massive graviton can play the role of a FIMP DM candidate for masses $M_{G} \lesssim$ a few $\mathrm{MeV}$. Note that the mass range makes the spin-2 massive graviton an ideal sub-MeV DM particle, which is currently favored by the small scale galaxy structure [35-37]. We have applied this new result to an extension of the Randall-Sundrum model with three branes. Depending on the radion mass, only the first two KK gravitons can be long-lived at a cosmological scale, while the higher modes and radion decay very effectively into DM. Note that the radion was considered as a DM candidate in [38], but being difficult to account for a heavy relic density. We have also implicitly assumed that the pair production of massive gravitons is subdominant due to the gauge invariance of self-interactions. This is the first time that a light spin-2 mode, or a class of "glueball" interpolated by the conserved energy-momentum tensor of the strong dynamics from AdS/CFT correspondence [39-41], is shown to be a feasible DM candidate. We illustrated that there exists a sizable parameter space where the light massive gravitons can saturate the DM relic density, while escaping the stringent bounds on decaying DM from indirect detection and the $\mathrm{CMB}$.

H. C. and S. L. were supported by the National Research Foundation of Korea (NRF) grant funded by the Korea government (MEST) (No. NRF-2021R1A2C1005615). H.C. acknowledges the support of Tsung-Dao Lee Institute, SJTU where this project is initiated. G. C. and S. L also acknowledge support from the Campus-France STAR project "Higgs and dark matter connections."

* Corresponding author.

hcai@korea.ac.kr †.cacciapaglia@ipnl.in2p3.fr sjjlee@korea.ac.kr

[1] D. Y. Cheong, S. M. Lee, and S. C. Park, J. Cosmol. Astropart. Phys. 01 (2021) 032.

[2] N. Aghanim et al. (Planck Collaboration), Astron. Astrophys. 641, A6 (2020).

[3] G. Bertone and M. P. T. Tait, Nature (London) 562, 51 (2018).

[4] L. J. Hall, K. Jedamzik, J. March-Russell, and S. M. West, J. High Energy Phys. 03 (2010) 080.

[5] F. Elahi, C. Kolda, and J. Unwin, J. High Energy Phys. 03 (2015) 048.

[6] T. Han, J. D. Lykken, and R.-J. Zhang, Phys. Rev. D 59, 105006 (1999).

[7] P. Gondolo and G. Gelmini, Nucl. Phys. B360, 145 (1991).

[8] See Supplemental Material at http://link.aps.org/ supplemental/10.1103/PhysRevLett.128.081806 for the detail calculation to derive the amplitude squared for the single production of a massive graviton, that leads to Eq. (4), Eq. (5) and Eq. (6) in the main text.

[9] G. F. Giudice, R. Rattazzi, and J. D. Wells, Nucl. Phys. B544, 3 (1999).

[10] E. A. Mirabelli, M. Perelstein, and M. E. Peskin, Phys. Rev. Lett. 82, 2236 (1999).

[11] C. Cheung, G. Elor, and L. J. Hall, Phys. Rev. D 85, 015008 (2012).

[12] S. Hannestad, Phys. Rev. D 70, 043506 (2004).

[13] M. Quiros, in ICTP Summer School in High-Energy Physics and Cosmology (1999), arXiv:hep-ph/9901312.

[14] R. Essig, E. Kuflik, S. D. McDermott, T. Volansky, and K. M. Zurek, J. High Energy Phys. 11 (2013) 193.

[15] V. Poulin, J. Lesgourgues, and P. D. Serpico, J. Cosmol. Astropart. Phys. 03 (2017) 043.

[16] L. Randall and R. Sundrum, Phys. Rev. Lett. 83, 4690 (1999).

[17] L. Randall and R. Sundrum, Phys. Rev. Lett. 83, 3370 (1999).

[18] W. D. Goldberger and M. B. Wise, Phys. Rev. Lett. 83, 4922 (1999). 
[19] I. I. Kogan, S. Mouslopoulos, A. Papazoglou, and L. Pilo, Nucl. Phys. B625, 179 (2002).

[20] K. Agashe, P. Du, S. Hong, and R. Sundrum, J. High Energy Phys. 01 (2017) 016.

[21] H. Cai, arXiv:2109.09681.

[22] H. Cai, arXiv:2201.04053.

[23] S. J. Lee, Y. Nakai, and M. Suzuki, arXiv:2109.10938.

[24] H. Davoudiasl, J. L. Hewett, and T. G. Rizzo, Phys. Rev. Lett. 84, 2080 (2000).

[25] H. Davoudiasl, J. L. Hewett, and T. G. Rizzo, Phys. Rev. D 63, 075004 (2001).

[26] G. F. Giudice, R. Rattazzi, and J. D. Wells, Nucl. Phys. B595, 250 (2001).

[27] C. Csaki, J. Hubisz, and S. J. Lee, Phys. Rev. D 76, 125015 (2007).

[28] E. Babichev, L. Marzola, M. Raidal, A. Schmidt-May, F. Urban, H. Veermäe, and M. von Strauss, J. Cosmol. Astropart. Phys. 09 (2016) 016.

[29] B. Bellazzini, C. Csaki, J. Hubisz, J. Serra, and J. Terning, Eur. Phys. J. C 73, 2333 (2013).
[30] Z. Chacko and R. K. Mishra, Phys. Rev. D 87, 115006 (2013).

[31] Z. Chacko, R. K. Mishra, and D. Stolarski, J. High Energy Phys. 09 (2013) 121.

[32] H. M. Lee, M. Park, and V. Sanz, Eur. Phys. J. C 74, 2715 (2014).

[33] B. M. Dillon, C. Han, H. M. Lee, and M. Park, Int. J. Mod. Phys. A 32, 1745006 (2017).

[34] H. Davoudiasl and T. G. Rizzo, Phys. Lett. B 512, 100 (2001).

[35] M. Boylan-Kolchin, J. S. Bullock, and M. Kaplinghat, Mon. Not. R. Astron. Soc. 415, L40 (2011).

[36] K. A. Oman etal., Mon. Not. R. Astron. Soc. 452, 3650 (2015).

[37] C. Dvorkin, T. Lin, and K. Schutz, Phys. Rev. Lett. 127, 111301 (2021)

[38] E. W. Kolb, G. Servant, and T. M. P. Tait, J. Cosmol. Astropart. Phys. 07 (2003) 008.

[39] J. M. Maldacena, Adv. Theor. Math. Phys. 2, 231 (1998).

[40] E. Witten, Adv. Theor. Math. Phys. 2, 253 (1998).

[41] S. S. Gubser, I. R. Klebanov, and A. M. Polyakov, Phys. Lett. B 428, 105 (1998). 